\title{
Vibration of Yukawa Potential Dependent Time and Extended Klein-Gordon Equation in Rindler Space-Time
}

\author{
Sangwha-Yi* \\ Department of Math, Taejon University 300-716, South Korea
}

*Corresponding Author: Sangwha-Yi, Department of Math, Taejon University 300-716, South Korea

\begin{abstract}
Atom's nucleus force understand by Yukawa potential independent time. We study Yukawa potential dependent about time. We make Klein-Gordon equation is satisfied by Yukawa potential dependent about time. Yukawa potential satisfy Proca equation or Klein-Gordon equation. If we represent Yukawa potential dependent time in Rindler space-time, this Yukawa potential satisfy the extended Klein-Gordon equation in Rindler space-time. We understand Yukawa force in Rindler space-time.
\end{abstract}

Keywords: Nucleus vibration; Yukawa potential; Klein-Gordon equation Rindler Space-time; Extended Klein-Gordon equation Yukawa force

PACS Number: 03.30. $+p, 03.65$

\section{INTRODUCTION}

Atom's nucleus force understand by Yukawa potential. We study Yukawa potential dependent about time. We make Klein-Gordon equation is satisfied by Yukawa potential dependent about time.

At first, Yukawa potential $V$ describes nucleus's combine force in semi-classical method.[7]

$V=-\frac{g^{2}}{r} \exp \left(-\frac{m_{\pi} r C}{\hbar}\right)$

$g$ is real number, $m_{\pi}$ is the meson's mass

Klein-Gordon equation is satisfied by Yukawa potential $V$.

$$
\begin{aligned}
& -\partial_{i} \partial V+\frac{m^{2} C^{2}}{\hbar^{2}} V=-\nabla^{2} V+\frac{m_{\pi}^{2} C^{2}}{\hbar^{2}} V=0 \\
& V=-\frac{g^{2}}{r} \exp \left(-\frac{m_{\pi} r C}{\hbar}\right)
\end{aligned}
$$

If we focus Klein-Gordon equation make 4-dimential partial differential equation about Yukawa potential $\phi$ dependent time,

$\frac{m_{\pi}^{2} C^{2}}{\hbar^{2}} \phi+\partial_{\mu} \partial^{\mu} \phi=\frac{m_{\pi}^{2} C^{2}}{\hbar^{2}} \phi+\frac{1}{C^{2}} \frac{\partial^{2}}{\partial t^{2}} \phi-\nabla^{2} \phi=0$

In this time, Yukawa potential $\phi$ dependent time is.

$$
\phi=-\frac{g^{2}}{r} \exp \left(-\frac{m_{\pi} r C}{\hbar}\right)+A_{0} \sin \omega t, \text { Frequency } \omega=\frac{m_{\pi} C^{2}}{\hbar}
$$


Absolutely, if we calculate, $\mathrm{Eq}(3)$ is satisfied by $\mathrm{Eq}(4)$. Yukawa potential $\phi$ is vibrated about the amplitude $A_{0}$, but we know the nuclear strong force doesn't vibrate about time in inertial frame..

2. Yukawa Potential DePendent Time from EXTENDEd KLEIN-Gordon EQUATION IN RINDLER-SPACE-TIME

Rindler coordinates are

$$
\begin{aligned}
& c t=\left(\frac{c^{2}}{a_{0}}+\xi^{1}\right) \sinh \left(\frac{a_{0}}{c} \xi^{0}\right), x=\left(\frac{c^{2}}{a_{0}}+\xi^{1}\right) \cosh \left(\frac{a_{0}}{c} \xi^{0}\right)-\frac{c^{2}}{a_{0}} \\
& y=\xi^{2}, z=\xi^{3}
\end{aligned}
$$

If we write Yukawa potential $\phi$ in inertial frame,

$\phi=-\frac{g^{2}}{r} \exp \left(-\frac{m_{\pi} r C}{\hbar}\right)+A_{0} \sin \omega t, \quad$ Frequency $\omega=\frac{m_{\pi} C^{2}}{\hbar}$

If we rewrite Yukawa potential $\phi_{\xi}$ in Rindler space-time,

$$
\begin{aligned}
& \phi=\phi^{1}+\phi^{2}=\phi_{\xi}=\phi_{\xi}{ }^{1}+\phi_{\xi}{ }^{2} \\
& \phi^{1}=\phi_{\xi}{ }^{1}=-\frac{g^{2}}{\sqrt{x^{2}+y^{2}+z^{2}}} \exp \left(-\frac{m_{\pi} C}{\hbar} \sqrt{x^{2}+y^{2}+z^{2}}\right) \\
& =-\frac{g^{2}}{\sqrt{\left\{\left(\frac{c^{2}}{a_{0}}+\xi^{1}\right) \cosh \left(\frac{a_{0}}{C} \xi^{0}\right)-\frac{c^{2}}{a_{0}}\right\}^{2}+\left(\xi^{2}\right)^{2}+\left(\xi^{3}\right)^{2}}} \exp \left[-\frac{m_{\pi} c}{\hbar} \sqrt{\left.\left\{\left(\frac{c^{2}}{a_{0}}+\xi^{1}\right) \cosh \left(\frac{a_{0}}{C} \xi^{0}\right)-\frac{c^{2}}{a_{0}}\right\}^{2}+\left(\xi^{2}\right)^{2}+\left(\xi^{3}\right)^{2}\right]}\right.
\end{aligned}
$$

And,

$$
\phi^{2}=\phi_{\xi}^{2}=A_{0} \sin \omega t=A_{0} \sin \left[\omega\left\{\left(\frac{c}{a_{0}}+\frac{\xi^{1}}{C}\right) \sinh \left(\frac{a_{0} \xi^{0}}{c}\right)\right\}\right]
$$

This Yukawa potential satisfy the extended Klein-Gordon equation. At first, energy and momentum are in Rindler space-time[1],

$$
E_{\xi}=i \hbar \frac{1}{\left(1+\frac{a_{0}}{c^{2}} \xi^{1}\right)} \frac{\partial}{\partial \xi^{0}}, \vec{p}_{\xi}=-i \hbar \vec{\nabla}_{\xi}
$$

Energy-Momentum equation is in Rindler space-time[1],

$$
E_{\xi}^{2}=\vec{p}_{\xi} c \cdot \vec{p}_{\xi} c+m^{2} c^{4}
$$

Hence, normal Klein-Gordon equation is in Rindler-spacetime,

$$
\frac{m_{\pi}^{2} C^{2}}{\hbar^{2}} \phi_{\xi}+\frac{1}{c^{2}} \frac{1}{\left(1+\frac{a_{0}}{c^{2}} \xi^{1}\right)^{2}} \frac{\partial^{2}}{\left(\partial \xi^{0}\right)^{2}} \phi_{\xi}-\nabla_{\xi}^{2} \phi_{\xi}=0
$$

In this time, we focus the gauge $\Lambda$ equation in Rindler space-time[1], 


$$
\frac{1}{c^{2}} \frac{1}{\left(1+\frac{a_{0}}{c^{2}} \xi^{1}\right)^{2}} \frac{\partial^{2}}{\left(\partial \xi^{0}\right)^{2}} \Lambda-\nabla_{\xi}^{2} \Lambda-\frac{\partial \Lambda}{\partial \xi^{1}} \frac{a_{0}}{c^{2}} \frac{1}{\left(1+\frac{a_{0}}{c^{2}} \xi^{1}\right)}=0
$$

Hence, Eq(12) change extended Klein-Gordon equation in Rindler space-time.

Extended Klein-Gordon Equation is in Rindler space-time,

$$
\frac{m_{\pi}{ }^{2} C^{2}}{\hbar^{2}} \phi_{\xi}{ }^{1}+\frac{1}{c^{2}} \frac{1}{\left(1+\frac{a_{0}}{c^{2}} \xi^{1}\right)^{2}} \frac{\partial^{2} \phi_{\xi}{ }^{1}}{\left(\partial \xi^{0}\right)^{2}}-\nabla_{\xi}{ }^{2} \phi_{\xi}{ }^{1}-\frac{\partial \phi_{\xi}{ }^{1}}{\partial \xi^{1}} \frac{a_{0}}{c^{2}} \frac{1}{\left(1+\frac{a_{0}}{c^{2}} \xi^{1}\right)}=0
$$

And

$\frac{m_{\pi}{ }^{2} C^{2}}{\hbar^{2}} \phi_{\xi}{ }^{2}+\frac{1}{c^{2}} \frac{1}{\left(1+\frac{a_{0}}{c^{2}} \xi^{1}\right)^{2}} \frac{\partial^{2} \phi_{\xi}^{2}}{\left(\partial \xi^{0}\right)^{2}}-\nabla_{\xi}{ }^{2} \phi_{\xi}{ }^{2}-\frac{\partial \phi_{\xi}{ }^{2}}{\partial \xi^{1}} \frac{a_{0}}{c^{2}} \frac{1}{\left(1+\frac{a_{0}}{c^{2}} \xi^{1}\right)}=0$

Hence,

$$
\frac{m_{\pi}^{2} C^{2}}{\hbar^{2}} \phi_{\xi}+\frac{1}{c^{2}} \frac{1}{\left(1+\frac{a_{0}}{c^{2}} \xi^{1}\right)^{2}} \frac{\partial^{2} \phi_{\xi}}{\left(\partial \xi^{0}\right)^{2}}-\nabla_{\xi}^{2} \phi_{\xi}-\frac{\partial \phi_{\xi}}{\partial \xi^{1}} \frac{a_{0}}{c^{2}} \frac{1}{\left(1+\frac{a_{0}}{c^{2}} \xi^{1}\right)}=0
$$

$\mathrm{Eq}(8), \mathrm{Eq}(9)$, Yukawa potentials $\phi_{\xi}{ }^{1}, \phi_{\xi}{ }^{2}$ satisfy $\mathrm{Eq}(14), \mathrm{Eq}(15)$, extended Klein-Gordon equations in Rindler space-time. Therefore, Eq(7), Yukawa potential $\phi_{\xi}$ satisfy Eq(16), extended KleinGordon equation in Rindler space-time

Yukawa force $\vec{f}$ is

$$
\vec{f}=-\vec{\nabla} \phi=-\frac{g^{2}}{r^{3}}\left[\exp \left(-\frac{m_{\pi} r c}{\hbar}\right)\right]\left(1+\frac{m_{\pi} r c}{\hbar}\right) \vec{r}
$$

In this time, Yukawa force $\vec{f}_{\xi}$ is Rindler space-time,

$$
\begin{aligned}
& \vec{f}_{\xi}=-\vec{\nabla}_{\xi} \phi_{\xi}=-\vec{\nabla}_{\xi} \phi_{\xi}{ }^{1}-\vec{\nabla}_{\xi} \phi_{\xi}{ }^{2}=-\frac{g^{2}}{r^{3}}\left[\exp \left(-\frac{m_{\pi} r c}{\hbar}\right)\right]\left(1+\frac{m_{\pi} r c}{\hbar}\right)\left(x \cosh \left(\frac{a_{0} \xi^{0}}{c}\right), \xi^{2}, \xi^{3}\right) \\
& -\frac{\omega}{C} A[\cos (\omega t)]\left(\sinh \left(\frac{a_{0} \xi^{0}}{C}\right), 0,0\right)
\end{aligned}
$$

Hence, according to Yukawa force $\vec{f}_{\xi}$ in Rindler space-time, the nuclear force strongly acts and vibrates in accelerated frame rather than inertial frame in $\mathrm{x}$-axis.

\section{CONCLUSION}

We found Yukawa potential dependent time. Hence, the nuclear strong force vibrates about time in Rindler spacetime. We found Yukawa potential mechanism in Rindler Space-time. We understand nuclear force in Rindler space-time. 
Vibration of Yukawa Potential Dependent Time and Extended Klein-Gordon Equation in Rindler SpaceTime

\section{REFERENCES}

[1]S.Yi, "Electromegnetic Wave Function and Equation, Lorentz Force in Rindler Space-time", International Journal of Advanced Research in Physical Science,5,9(2018)

[2]J.M. Normand, A Lie group: Rotations in Quantum Mechanics( North-Holland Pub. Co., 1980)

[3]J.D. Bjorken \& S. D. Drell, Relativistic Quantum Field(McGraw- Hill Co., 1965)

[4]P.Bergman,Introduction to the Theory of Relativity(Dover Pub. Co.,Inc., New York,1976),Chapter V

[5]R.L.Liboff, Quantum Mechanics(Addison-Wesley Publishing Co., Inc.,1990)

[6]A.Beiser, Concept of Modern Physics(McGraw-Hill,Inc.,1991)

[7]H. Yukawa, "On the interaction of elementary particle. I", Proceedings of the Physico-Mathematical Society of Japan. $3^{\text {rd }}$ Series 17(1935):48-573

Citation: Sangwha-Yi, (2020). "Vibration of Yukawa Potential Dependent Time and Extended KleinGordon Equation in Rindler Space-Time". International Journal of Advanced Research in Physical Science (IJARPS) 7(7), pp.1-4, 2020.

Copyright: () 2020 Authors, This is an open-access article distributed under the terms of the Creative Commons Attribution License, which permits unrestricted use, distribution, and reproduction in any medium, provided the original author and source are credited. 